\title{
Buruli Ulcer in Japan
}

\author{
Koichi Suzuki, Yuqian Luo, Yuji Miyamoto, Chiaki Murase, \\ Mariko Mikami-Sugawara, Rie R. Yotsu, and Norihisa Ishii
}

\section{$1 \quad$ Epidemiology and Bacteriological and Genomic Features of $M$. ulcerans subsp. shinshuense}

\section{$1.1 \quad$ Epidemiology}

Japan is one of the few countries located in a temperate zone that reports cases of Buruli ulcer (BU). The first case, a 19-year-old female who presented with a chronic and necrotic ulcer on her left elbow, was reported by Mikoshiba et al. in 1982 [1]. This was considered to be an endemic infection due to the lack of a travel history outside the country. A taxonomic study using DNA hybridization assays was later performed on a mycobacterial strain (ATCC 33788) isolated from the skin ulcer lesion of this patient, revealing that this Japanese strain was highly similar to the classical Mycobacterium ulcerans strain ATCC 19423. However, the Japanese strain differed from the previously described M. ulcerans strain in mycolic acid

K. Suzuki · Y. Luo

Department of Clinical Laboratory Science, Teikyo University, Tokyo, Japan

e-mail: koichis0923@med.teikyo-u.ac.jp

Y. Miyamoto $\cdot$ N. Ishii $(\triangle)$

Leprosy Research Center, National Institute of Infectious Diseases, Tokyo, Japan

e-mail: yujim@nih.go.jp; norishii@niid.go.jp

C. Murase

Department of Dermatology, Nagoya University Graduate School of Medicine,

Nagoya, Japan

M. Mikami-Sugawara

West Yokohama Sugawara Dermatology Clinic, Yokohama, Japan

Department of Environmental Immuno-Dermatology, Yokohama City University Graduate

School of Medicine, Yokohama, Japan

R. R. Yotsu

National Sanatorium Suruga, Shizuoka, Japan

(C) The Author(s) 2019

G. Pluschke, K. Röltgen (eds.), Buruli Ulcer,

https://doi.org/10.1007/978-3-030-11114-4_5 
composition and some biological and biochemical characteristics; therefore, it was deemed to belong to a new subspecies of M. ulcerans named M. ulcerans subsp. shinshuense [2]. The disease was not noticed again in Japan until the second case of BU was identified and reported in 2003 [3]. Since then, the number of cases has increased gradually, summing to a total of 60 cases reported from 17 of the country's 47 prefectures till the end of 2016. M. ulcerans subsp. shinshuense has been isolated from 41 of the cases [4-7].

BU cases are rather sporadically distributed throughout the main and largest island of Japan, Honshu Island, with no particular focus [6, 8]. The Okayama Prefecture, which is localized in the central western regions of Japan facing the Seto Sea, has so far reported most of the cases (ten [17\%] cases), followed by the Tottori Prefecture (six [10\%] cases) and the Shiga Prefecture (seven [12\%] cases). The Akita Prefecture is the most northern prefecture reporting BU cases, where the temperature can be below zero degrees Celsius during the coldest season of the year. All reported cases in Japan are considered to be domestic infections as none of the patients had a history of oversea travel to BU endemic countries before symptom onset. The acquisition of the pathogen from a suspicious aquatic environment remains undetermined in most cases. However, it is interesting to note that most BU cases in Japan were diagnosed in autumn and winter (48 cases), which may indicate a higher incidence of infection during summer considering the expected time lag between infection and symptom onset [9].

\subsection{Genome}

M. ulcerans and related mycobacterial strains (such as $M$. liflandii) share a characteristic genome structure consisting of a chromosome and a giant plasmid. This genome structure caused some difficulty when analyzing the whole genome sequences. Recently, with the help of next-generation sequencing (NGS), the complete genome sequences of two representative isolates, M. ulcerans Agy99 and $M$. liflandii 128FXT, were unraveled [10-12]. The complete genome sequence of $M$. ulcerans subsp. shinshuense (ATCC 33728) was also reported by a Japanese group [13]. Comparison of the whole genome of M. ulcerans subsp. shinshuense with those of the related strains revealed that it bears more similarity with $M$. liflandii 128FXT than with M. ulcerans Agy99 (Table 1). M. ulcerans subsp. shinshuense has a 5,899,681 bp chromosome with $65.64 \%$ GC content and a 166,617 bp giant plasmid with $62.76 \%$ GC content. The average nucleotide identities were $98.36 \%$ to M. ulcerans Agy99 and 99.10\% to M. liflandii 128FXT [13]. The total number of coding DNA sequences (CDSs) in both M. ulcerans subsp. shinshuense and $M$. liflandii 128FXT were found to be roughly around 5000, approximately $800 \mathrm{CDSs}$ more than in the M. ulcerans Agy99 chromosome (Table 1) [10-13]. With respect to insertion sequences (IS), IS2606 was much more abundant in M. ulcerans Agy99 than in M. ulcerans subsp. shinshuense and M. liflandii 128FXT (Table 1) [10-13]. In contrast, more than 200 copies of IS2404 were observed in M. ulcerans subsp. shinshuense, which share approximately $99 \%$ identity with the classical $M$. 
Table 1 Genomic characteristics of M. ulcerans subsp. shinshuense and related mycobacteria

\begin{tabular}{|c|c|c|c|}
\hline Characteristics & $\begin{array}{l}\text { M. ulcerans subsp. shinshuense ATCC } \\
33728\end{array}$ & $\begin{array}{l}\text { M. ulcerans } \\
\text { Agy99 }\end{array}$ & \begin{tabular}{|l} 
M. liflandii \\
128FXT
\end{tabular} \\
\hline \multicolumn{4}{|l|}{ Chromosome } \\
\hline Size (bp) & $5,899,681$ & $5,631,606$ & $6,208,955$ \\
\hline $\mathrm{G}+\mathrm{C}(\%)$ & 65.64 & 65.47 & 65.62 \\
\hline No. of CDSs & 5015 & 4160 & 4994 \\
\hline $\begin{array}{l}\text { No. of } \\
\text { pseudogenes }\end{array}$ & 451 & 771 & 436 \\
\hline No. of IS2404 & 206 & 209 & 224 \\
\hline No. of IS 2606 & 1 & 83 & 1 \\
\hline \multicolumn{4}{|l|}{ Plasmid } \\
\hline Size (bp) & 166,617 & 174,155 & 190,588 \\
\hline $\mathrm{G}+\mathrm{C}(\%)$ & 62.76 & 62.5 & 62.9 \\
\hline No. of CDSs & 72 & 72 & 95 \\
\hline $\begin{array}{l}\text { No. of } \\
\text { pseudogenes }\end{array}$ & 7 & 7 & 22 \\
\hline No. of IS2404 & 4 & 4 & 15 \\
\hline No. of IS2606 & 1 & 8 & 3 \\
\hline References & [7], Yoshida et al. (unpublished) & [8] & {$[9,10]$} \\
\hline $\begin{array}{l}\text { GenBank accession } \\
\text { no. }\end{array}$ & $\begin{array}{l}\text { AP017624 } \\
\text { AP017625 }\end{array}$ & $\begin{array}{l}\text { CP000325 } \\
\text { BX649209 }\end{array}$ & $\begin{array}{l}\text { NC_020133 } \\
\text { NC_011355 }\end{array}$ \\
\hline
\end{tabular}

$C D S$ coding DNA sequence, $I S$ insertion sequence

Table 2 Nuclotide substitutions observed in 16S rRNA sequences from M. ulcerans subsp. shinshuense and related mycobacteria

\begin{tabular}{|c|c|c|c|}
\hline \multirow[b]{2}{*}{ Species } & \multicolumn{2}{|c|}{$\begin{array}{l}\text { Differentiating sequence position } \\
\text { (underline) }\end{array}$} & \multirow{2}{*}{$\begin{array}{l}\text { GenBank } \\
\text { accession no. }\end{array}$} \\
\hline & 492 & 1288 & \\
\hline $\begin{array}{l}\text { M. ulcerans subsp. shinshuense } \\
\text { ATCC } 33728\end{array}$ & TGGGGAA & TAA $\underline{G G C C}$ & AB548733 \\
\hline M. ulcerans Agy99 & TGGAGGAA & TAA $\underline{C G C C}$ & AB548729 \\
\hline M. liflandii $128 \mathrm{FXT}$ & TGG $\underline{\mathbf{A} G A A}$ & TAA $\underline{\mathbf{A} G C C}$ & CP003899 \\
\hline M. marinum ATCC 927 & TGGÁGAA & TAA $\underline{\mathbf{A} G C C}$ & AB548717 \\
\hline M. pseudoshottsii JCM 15466 & TGGㅁGAA & TAA $\underline{\mathbf{A} G C C}$ & AB548713 \\
\hline
\end{tabular}

ulcerans strains and M. liflandii $[4,7,13]$. Due to the abundance of IS2404, PCR amplification of a partial IS2404 sequence is currently used for sensitive detection of M. ulcerans subsp. shinshuense, although differentiation from other closelyrelated IS2404-harboring species requires further investigation. The 16S rRNA sequences in $M$. ulcerans subsp. shinshuense were almost identical to those in $M$. ulcerans Agy99 (Table 2), except for two characteristic nucleotide substitutions at base positions 492 and 1288 found exclusively in M. ulcerans subsp. shinshuense (Table 2) [4, 7]. Including the listed representative isolate ATCC 33728 (Table 2), the same nucleotide substitutions have been found in ten clinical isolates of $M$. ulcerans subsp. shinshuense [7]. 
Comparison of eight pMUM001 gene sequences encoding lipid toxin mycolactoneproducing enzymes (including repA, parA, serine/threonine protein kinase [STPK] gene, loading domain of $m l s$, acyltransferase domain of $m l s$, rep type II thioesterase gene, rep type III ketosynthase gene, and rep $\mathrm{P} 450$ hydroxylase gene) in the giant plasmid of M. ulcerans subsp. shinshuense and the African M. ulcerans strain by PCR amplification revealed the presence of seven pMUM001 gene sequences and the loss of the STPK gene in Japanese isolates $[4,7]$. The elucidation of the complete genome sequence of M. ulcerans subsp. shinshuense further verified the above finding by showing that the STPK gene in M. ulcerans subsp. shinshuense is truncated, with low similarity to STPK in M. ulcerans Agy99 [13]. Despite the deficiency of a functional STPK gene in Japanese clinical isolates, no apparent bacteriological differences were observed, indicating the possibility that some signaling pathways compensate for STPK function in M. ulcerans subsp. shinshuense [14]. Intriguingly, a Chinese $M$. ulcerans strain has a $16 \mathrm{~S}$ rRNA gene identical in sequence to that in $M$. ulcerans subsp. shinshuense, and also lacks the STPK gene in its giant plasmid like M. ulcerans subsp. shinshuense [4, 7]. This finding supports the suggestion that the Chinese strain is phylogenetically closest to M. ulcerans subsp. shinshuense among other M. ulcerans strains [15]. However, further characterization of the whole genome sequence of the Chinese strain is required for its classification.

The close resemblance, in addition to distinctive genomic features, between the classical M. ulcerans strains and the Japanese strain M. ulcerans subsp. shinshuense has evoked great interest in the intra-species evolutionary scenario for $M$. ulcerans. Phylogenetic analysis of irreversible genomic changes focusing on insertion-deletion polymorphisms in 12 regions of difference among $M$. ulcerans strains has unambiguously resolved a phylogenetic tree showing that $M$. ulcerans has evolved into at least two distinct lineages since divergence from the M. marinum progenitor [15]. In addition to M. ulcerans subsp. shinshuense, strains from China, South America, and Mexico were shown to belong to a lineage that is more closely related to M. marinum, namely the 'ancestral' lineage; while strains from Africa and Australia belong to the 'classical' lineage that has undergone major genomic rearrangement [15]. In agreement with this evolutionary scenario, 26,564 single nucleotide polymorphisms (SNPs) were found in a Japanese strain by a comparison with the reference genome of the classical lineage isolate Agy99 using a high-resolution phylogeny analysis based on genome-wide SNPs [16]. Moreover, calculation of time scales of the evolutionary process by the minimum evolution tree-based approach estimated that the divergence of Ghanaian subtypes (including Agy99) from the M. marinum progenitor occurred about 1000-3000 years ago, whereas M. ulcerans subsp. shinshuense diverged much earlier (about 394,000-529,000 years ago) [16].

\subsection{Biochemical Properties}

Mycolactone, secreted by M. ulcerans in the process of chronic infection, is an essential molecule for the virulence of BU [17]. Mycolactone is also secreted by strains originally designated $M$. pseudoshottsii, M. liflandii, and a subset of M. marinum strains [18-20]. M. ulcerans subsp. shinshuense produces mycolactone $\mathrm{A} / \mathrm{B}$, among 


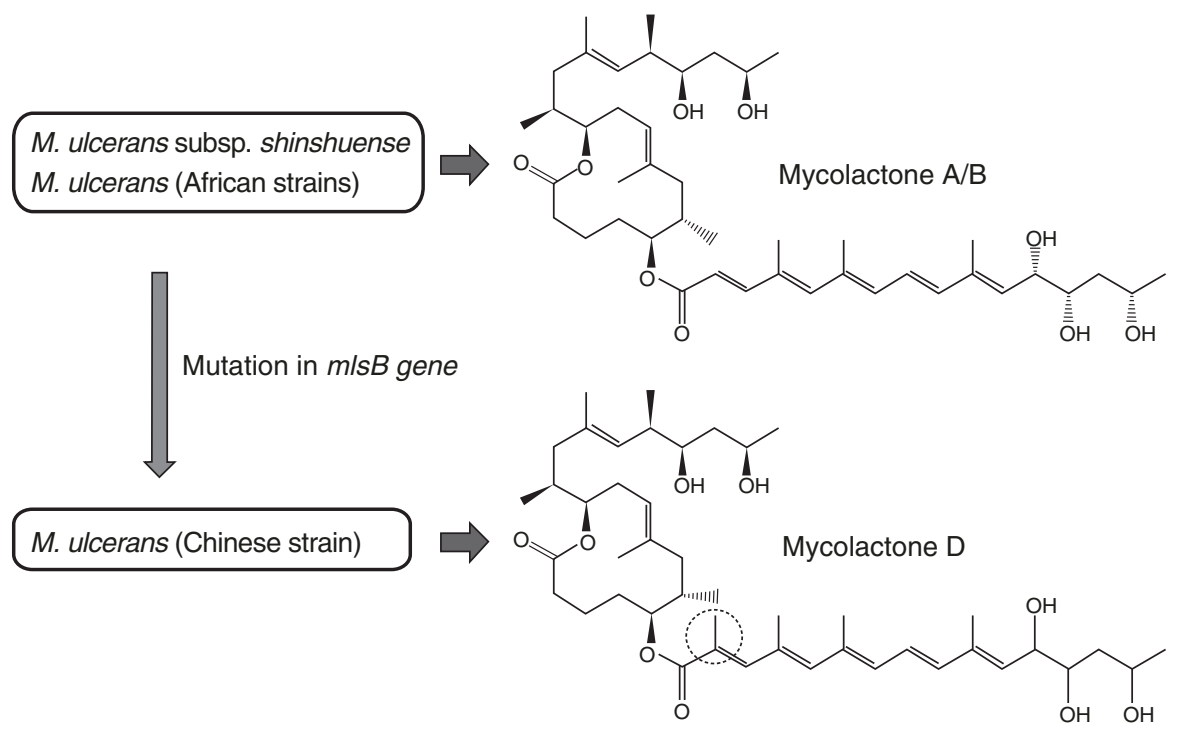

Fig. 1 Structural variation in mycolactones caused by a $m l s B$ mutation uniquely found in a Chinese $M$. ulcerans strain. The dashed circle in mycolactone D represents the methyl group that distinctively differentiates it from mycolactone A/B produced by M. ulcerans subsp. shinshuense and African strains of $M$. ulcerans

five analogs of mycolactone, which was originally found in African M. ulcerans strains [12, 17, 18] (Fig. 1). In contrast, the Chinese strain of M. ulcerans produces a unique mycolactone $\mathrm{D}$ that is not observed in other M. ulcerans strains [21]. Mycolactone $\mathrm{D}$ possesses an additional methyl group in its acyl side chain, whereas the mycolactone A/B conserves an intact form (Fig. 1) [12, 17, 18, 21]. In the Chinese strain, nonsynonymous substitutions that could cause drastic functional changes have been found in the $m l s B$ gene involved in synthesis of acyl side chains, while corresponding mutations were not observed in Japanese M. ulcerans subsp. shinshuense or the African strains, suggesting that the Chinese strain might have uniquely acquired the ability to modify mycolactone $\mathrm{A} / \mathrm{B}$ to $\mathrm{D}$ through genetic alternations [12].

A variety of glycolipids are present in the mycobacterial cell wall that likely functions as a protective barrier from host immune attack [22, 23]. Mycolate is a type of long chain fatty acid covalently linked to various glycolipids as a major component in the mycobacterial cell wall [22, 23]. Mycobacteria produce three main types of mycolate: alpha-, keto- (which contains additional ketone groups), and methoxy-mycolate (which contains additional methoxy groups) [22, 23]. Tsukamura et al. reported that both $M$. ulcerans subsp. shinshuense and other $M$. ulcerans strains share the three types of mycolate, while the average number of carbons in mycolate in M. ulcerans subsp. shinshuense is higher than that in an Australian M. ulcerans strain (ATCC 19423) [2]. However, comprehensive comparison is needed to determine whether the distribution of the three mycolate types differs between $M$. ulcerans subsp. shinshuense and other M. ulcerans strains, and to unravel their implications in mycobacterial virulence. 
As one major glycolipid component in the mycobacterial cell wall, phenolic glycolipids (PGLs) were found in M. leprae, M. tuberculosis, M. marinum, and $M$. ulcerans [24-26]. PGLs vary in their compositions of sugar moieties (including rhamnose, fucose, and glucose), which are highly species-specific [24-26]. Daffe et al. found that $10 \%$ of $M$. ulcerans strains produced PGL consisting of monosaccharide (3-O-methyl-rhamnose), supporting a close phylogenetic link between $M$. ulcerans and M. marinum [27]. However, the rest of the tested strains, including $M$. ulcerans subsp. shinshuense, did not produce PGL [27]. In M. leprae, PGL is known to induce a strong antibody response and to play a role in invasion into Schwann cells [28, 29]. In M. tuberculosis, clinical isolates have been divided into PGLproducing and non-producing groups, and the former is regarded as more virulent, suggesting that PGL plays a role in the pathogenicity of mycobacteria [30]. This suggests that PGL-producing M. ulcerans subsp. shinshuense strains may be found, albeit less frequently. Analysis of PGL in M. ulcerans subsp. shinshuense as well as in other $M$. ulcerans strains with reference to the clinical symptoms in each case might offer a new approach to understand the causes of invasive BU cases.

Drug susceptibility tests showed that $M$. ulcerans subsp. shinshuense strains were more sensitive to streptomycin (SM), kanamycin (KM), and clarithromycin (CAM) than reference M. ulcerans strains (ATCC 19423 and Agy99) [7]. Differing from other antimicrobial drugs, such as rifampicin (RFP) and levofloxacin (LVFX), in the mechanism of action, binding to ribosomal RNA is exclusively required for SM, KM, and CAM to exert their actions to inhibit mycobacterial protein synthesis. The specific nucleotide substitutions found in the 16S rRNA of M. ulcerans subsp. shinshuense might have affected the conformation of its transcribed ribosomal structure, rendering it more accessible to the drugs $[4,7]$.

M. ulcerans subsp. shinshuense strains and the Chinese strain were all positive for urease activity, whereas the M. ulcerans strains from Africa and Australia were negative [7]. This feature may allow scientists to distinguish M. ulcerans subsp. shinshuense from $M$. ulcerans strains by a simple urease activity test. It has been shown in some mycobacterial species that urease plays a key role in metabolizing urea as a sole nitrogen source under nutrition-limited conditions and in neutralizing the surrounding milieu to inhibit phagolysosomal maturation [31, 32], indicating that urease-positive strains may be more adapted to harsh environments and more capable of achieving intracellular survival. Further comparisons between urease-positive strains with urease-deficient mutants might clarify whether urease activity contributes to the persistence of $M$. ulcerans subsp. shinshuense in the host.

\section{Clinical Features and Treatment of BU in Japan}

\subsection{Antimicrobial Treatment}

In the BU cases diagnosed in Japan, skin lesions mostly developed on exposed body parts including upper and lower limbs, face, ear, jaw, and clavicle. Although multiple lesions were present in 14 cases, no single lesion larger than $5 \mathrm{~cm}$ in diameter 
was reported. Therefore, all cases fall into World Health Organization (WHO) category I or II. Advanced BU infection has not been seen in Japan. In contrast to the general impression that BU lesions are typically painless, approximately half of the cases confirmed to be infected by M. ulcerans subsp. shinshuense have reported pain $[5,8]$.

In Japan, a variety of antimicrobial drugs have been used to treat BU; e.g., RFP, minocycline (MINO), CAM, LVFX, gatifloxacin (GFXN), norfloxacin (NFX), ethambutol (EB), isoniazid (INH), ethionamide (ETH), and tosufloxacin (TFLX). However, surgical intervention was often unavoidable. Nakanaga et al. tested the drug susceptibilities of $M$. ulcerans subsp. shinshuense to major antimicrobial agents and found that it is most susceptible to RFP and CAM, while resistant to EB, ETH, and INH (Table 3) [7]. LVFX, a broad-spectrum fluoroquinolone antibiotic, which also showed satisfactory activity against M. ulcerans and M. ulcerans subsp. shinshuense (Table 3) [7], has been preferentially used for treating skin infections in Japan due to its better transferability to soft tissue and skin. Based on these reasons and in reference to WHO BU guidelines, a triple antimicrobial therapy with RFP (450 mg/day), LVFX (500 mg/day), and CAM (800 mg/day), called RLC therapy, has been recommended and has increased cure rates of BU without surgery (Table 4).

In our latest investigation on the efficacy of BU treatment in Japan, 50 cases (83\% of the total reported cases) consisting of 19 male and 31 female patients, aged from 2 to 88 years (average: $45.8 \pm 27.63$ years) were included. Ten cases were excluded because of the absence of detailed information for treatment. These $50 \mathrm{BU}$ cases were divided in two groups based on their antimicrobial regimens. One group (21 cases) received highdose RLC (CAM at $800 \mathrm{mg} /$ day). The other 29 cases received other treatments including low-dose RLC (CAM at $400 \mathrm{mg}$ /day) or monotherapy of RFP, CAM or MINO; this group also included cases with interrupted RLC due to side effects and subsequent alternative chemotherapies. Five children received TFLX (12 mg/kg/day) substituted for LVFX, as TFLX (but not LVFX) is covered by medical insurance for children in Japan.

Table 3 Susceptibility testing results of M. ulcerans subsp. shinshuense

\begin{tabular}{l|l|l}
\hline \multirow{2}{*}{ Antimicrobial agent } & \multicolumn{2}{|l}{ MIC $(\mu \mathrm{g} / \mathrm{mL})$} \\
\cline { 2 - 3 } & M. ulcerans subsp. shinshuense \\
\cline { 2 - 3 } SM & ATCC33728 & 501 \\
\hline EB & 0.125 & 0.25 \\
\hline KM & 16 & 8 \\
\hline INH & 0.25 & 0.25 \\
\hline RFP & 8 & 8 \\
\hline LVFX & 0.06 & 0.06 \\
\hline CAM & 0.25 & 0.5 \\
\hline ETH & 0.03 & 0.06 \\
\hline AMK & 16 & 8 \\
\hline
\end{tabular}

$A M K$ amoxicillin, $C A M$ clarithromycin, $E B$ ethambutol, ETH ethionamide, $I N H$ isoniazid, $L V F X$ levofloxacin, $K M$ kanamycin, $M I C$ minimal inhibitory concentration, $R F P$ rifampicin, $S M$ streptomycin 
Table 4 Efficacy analysis of treatment for BU in Japan

\begin{tabular}{l|l|l|l|l|l|l}
\hline Treatment & No. & Age & $\begin{array}{l}\text { Sex } \\
\text { (M/F) }\end{array}$ & $\begin{array}{l}\text { Category } \\
\text { (I/II) }\end{array}$ & $\begin{array}{l}\text { Ulcer size } \\
(\mathrm{mm})\end{array}$ & $\begin{array}{l}\text { Cured completely } \\
\text { without surgery }\end{array}$ \\
\hline $\begin{array}{l}\text { RLC (high } \\
\text { dose) }\end{array}$ & 21 & $42.1 \pm 30.2$ & $11 / 10$ & $18 / 3$ & $25.6 \pm 23.8$ & 15 \\
\hline Others & 29 & $48.4 \pm 26.3$ & $8 / 21$ & $24 / 5$ & $27.3 \pm 15.7$ & 4 \\
\hline$P$ value & & 0.43 & 0.087 & 1.0 & 0.79 & $6.95 \times 10^{-5}$ \\
\hline
\end{tabular}

$B U$ Buruli ulcer, $R L C$ triple therapy with rifampicin ( $450 \mathrm{mg} /$ day), levofloxacin (500 $\mathrm{mg} /$ day), and clarithromycin $(800 \mathrm{mg} / \mathrm{day})$

Fifteen/twenty-one (71.4\%) high-dose RLC-treated patients compared to 4/29 (13.7\%) of the other patients had avoided surgery $(P<0.0001$, Fisher's exact test). The distribution of age, sex, size of skin ulcer, or WHO category did not significantly differ for patients treated with high-dose RLC therapy and the others. Together, these results suggest that high-dose RLC therapy has been most effective among all chemotherapies (Table 4), which is in agreement with a previous investigation that included $40 \mathrm{BU}$ cases in Japan by June 2013 [8]. The duration of high-dose RLC therapy ranged from 8 to 48 weeks ( 24 weeks in most cases), which was compared to the standard 8-week antibiotic treatment. The failure to determine a significant correlation between the duration of high-dose RLC therapy and the size of skin ulcers $(r=-0.2$, with Pearson correlation coefficient) indicates a tendency of overtreatment for BU in Japan.

The ineffectiveness of low-dose RLC may be attributed to a complex pharmacokinetic interaction between CAM and RFP. It has been reported that RFP induces production of cytochrome P450 3A4, an enzyme involved in the metabolism of CAM $[33,34]$, which would hasten the elimination of CAM from serum when used together $[35,36]$. These reports indicate the need for a higher dose of CAM when used in combination with RFP. With reference to the WHO BU meeting in 2015, we currently recommend a high-dose RLC triple therapy consisting of RFP $(10 \mathrm{mg} / \mathrm{kg} /$ day), LVFX (500 mg/day), and CAM (800 mg/day) in Japan [37].

\subsection{Alternative Treatments of BU}

Surgical excision and skin grafting were the only options for BU treatment until 2004 [38]. Since then, apart from chemotherapy also other therapeutic options, such as negative-pressure wound therapy (NPWT), ozone therapy, and hyperbaric oxygen therapy (HBOT) have been used or considered in the treatment of BU. Particularly for patients who have received surgical excision and still present large non-granulating wound areas, NPWT or ozone therapy could be a good choice for the next stage.

Since larger lesions or multiple ulcerative lesions often took a longer time to heal or failed to be cured by chemotherapy alone, surgical excision of the necrotic, ulcerated tissues had been performed in Japan. Among 31 cases that received surgical excision, $21(67.7 \%)$ also received skin grafts (Table 5). Among the 21 patients, NPWT was tentatively performed in one patient for the purpose of wound bed preparation before skin grafting [39]. This patient had an ulcer on the right ankle with a necrotic 
Table 5 Treatment for BU in Japan

\begin{tabular}{l|l|l|l}
\hline Treatment & $\begin{array}{l}\text { RLC (high dose) } \\
(\mathrm{n}=21)\end{array}$ & $\begin{array}{l}\text { Others } \\
(\mathrm{n}=29)\end{array}$ & $\begin{array}{l}\text { Total } \\
(\mathrm{n}=50)\end{array}$ \\
\hline Antimicrobial treatments only & 15 & 4 & 19 \\
\hline Surgical excision & 2 & 8 & 10 \\
\hline Surgical excision + skin graft & 3 & 17 & 20 \\
\hline $\begin{array}{l}\text { Surgical excision + NPWT + skin } \\
\text { graft }\end{array}$ & 1 & 0 & 1 \\
\hline BU Burgl & & & \\
\hline
\end{tabular}

$B U$ Buruli ulcer, NPWT negative-pressure wound therapy, $R L C$ triple therapy with rifampicin (450 mg/day), levofloxacin (500 mg/day), and clarithromycin ( $800 \mathrm{mg} /$ day)

bed, and the Achilles tendon and the calcaneal bone were exposed. NPWT with the Vacuum-Assisted Closure (V.A.C.) Therapy System (Kinetics Concepts Inc.; KCI) was started as a pretreatment for a skin graft to be performed 22 days after surgical excision of the necrotic tissue. The vacuum suction was maintained at $125 \mathrm{mmHg}$. The wound dressings were changed every third day for 24 days. At the end of NPWT, acceptable granulation tissue had covered the ulcer bed and the skin graft was consequently successfully engrafted. The patient was cured without disability.

NPWT has been widely used in Japan for many kinds of ulcerative lesions or for wound treatment after skin grafting, as it can reduce exudate, improve local edema, and activate granulation. It is not to be used for wounds with active infection. Therefore, for BU treatment, NPWT is preferable for use after surgical debridement combined with appropriate antimicrobial chemotherapy. Dressings must be changed every 48-72 $\mathrm{h}$. The absence of active wound infection should be checked each time at dressing. The exact frequency of dressing changes depends on the individual patient's circumstance, but it should not be less than three times a week. There are rental and purchasable NPWT (portable and disposable) models. Rental models include RENASYS by Smith \& Nephew and V.A.C. by KCI, and purchasable models include PICO by Smith \& Nephew. Otherwise healthy patients often must remain hospitalized for the sole purpose of receiving NPWT because the rental devices are only for hospital use. Thus, an affordable and purchasable NPWT device like PICO may be more cost-effective for an otherwise healthy patient. Nevertheless, its high cost is a large problem for patients in many countries (Table 6).

\subsection{Other Suggested Therapies}

Ozone therapy has been reported to shorten the course of wound healing and to promote lipid peroxidation and antioxidant protection indices [40]. Briefly, $\mathrm{O}_{3}$ is topically administered by positioning a bag around the lesion and insufflating an $\mathrm{O}_{2}-\mathrm{O}_{3}$ mixture at a concentration of $25-30 \mu \mathrm{g} / \mathrm{mL}$. To avoid gas leakage, the inflated bag is sealed just above the lesion. The bag is positioned and closed with an elastic band to give the gas mixture contact with the wound for about $20 \mathrm{~min}$. When the bag is removed, the wound is covered with a sterile gauze. A case with a skin ulcer in Benin was reported to be healed after the ozone applications in 2 weeks [41]. 
Table 6 Approximate costs for NPWT per week

\begin{tabular}{|c|c|c|c|}
\hline & \multicolumn{3}{|l|}{ Company and device } \\
\hline & \multicolumn{2}{|l|}{ Smith and Nephew } & $\mathrm{KCI}$ \\
\hline Country & RENASYS (US\$/week) & PICO (US\$/week) & V.A.C. (US\$/week) \\
\hline Japan & 620 & 420 & 700 \\
\hline South Africa (private) & N/A & N/A & 600 \\
\hline South Africa (public) & 450 & $175-300$ & 450 \\
\hline Egypt (private) & $290-340$ & 220 & N/A \\
\hline Egypt (public) & $145-155$ & N/A & N/A \\
\hline
\end{tabular}

Costs shown are approximate costs per week as of January 2017. The weekly costs include the costs for three sets of dressings and canisters and rental fee for the NPWT device (RENASYS and V.A.C.). The costs vary due to the size of lesions. $\mathrm{KCI}$ Kinetics Concepts Inc., N/A not available, $N P W T$ negative-pressure wound therapy, V.A.C. vacuum-assisted closure therapy system

Compared to surgical treatment, NPWT and ozone therapy are easy to use procedures. NPWT requires only the NPWT device, dressings, and canisters, though the disposable dressings and canisters are expensive. In contrast, ozone therapy does not require such expensive disposable materials. It requires an oxygen tank, an ozone generator, sterile water, and plastic bags, together with a system to drive the gas into contact with the lesions. The oxygen generator is expensive (4000-8000 Euros), but the cost of the disposable items for ozone therapy is much lower than that for NPWT. This simpler and more affordable therapy may offer an effective option for managing BU as an alternative treatment in endemic areas.

HBOT has long been established to treat skin ulcers and wounds such as diabetic foot ulcers, burns, and serious soft tissue infections including gas gangrene and necrotizing fasciitis [42]. HBOT is the application of $100 \%$ oxygen at a pressure two or three times of the atmospheric pressure at sea level. This pressure increases arterial and tissue oxygen tension to exert multiple physiological and therapeutic effects [43]. HBOT has been experimentally used to treat mice with M. ulceransinfected footpads $[44,45]$. In human patients, Pszolla et al. reported that supportive therapy with HBOT promoted healing after surgical excision of the deep ulcers with osteomyelitis caused by $M$. ulcerans [45]. HBOT requires hyperbaric chambers, which may limit its application in endemic areas. However, if used in conjunction with other therapeutic procedures, HBOT may be an effective therapeutic adjunct in treating BU, and it may contribute to reduce large surgical interventions like amputations and to prevent permanent disabilities [46].

\section{$3 \quad$ M. ulcerans subsp. shinshuense in the Environment of Japan}

\subsection{Detection of Environmental M. ulcerans subsp. shinshuense in Japan}

So far, M. ulcerans has been detected in the backyard of a family house in a rare case of concurrent familial clustering in Japan [47, 48]. In November, three members of a family living in a rural town in Japan, developed BU symptoms almost 
simultaneously. At first, the 2-year-old daughter had a gradually enlarging eruption on her right cheek. Soon after her symptom onset, an asymptomatic indurated eruption appeared on the right forearm of her 5-year-old brother, and a gradually enlarging eruption developed on the right wrist of her 37-year-old mother, both of which showed acid-fast bacilli-positive histopathological features similar to the lesions found in the little girl. The patients were tentatively given oral anti-mycobacterial multi-drug therapy combined with surgical treatment and cured without recurrence. PCR amplification and gene sequencing afterwards determined the strains isolated from the patients' skin biopsy cultures to be M. ulcerans subsp. shinshuense, thus giving an indubitable diagnosis of BU. The concurrent familial cases strongly suggested the presence of the pathogen in their common living environment, although person-to-person transmission of M. ulcerans cannot be excluded. A field study involving environmental sampling was therefore carried out in summer (July and August) and autumn (October) of the following year in an attempt to determine the presence of $M$. ulcerans in the environment around the family's house.

The family was living in an old house surrounded by rich farmland and extensive irrigation channels. Immediate suspicions centered on a one-meter-wide agricultural water channel of stagnant water slowly flowing through the family's backyard, to which the family had routine access and where the children entertained themselves by catching small water creatures. Environmental samples, including water, mud, crayfish, earthworms, a freshwater snail, a hoverfly, a moth, and several kinds of aquatic or semi-aquatic insects, were collected from or near the water channel in the patients' backyard in summer and autumn. Genomic DNA was purified from each sample and subjected to whole genome amplification (WGA) to achieve higher sensitivity in PCR to detect trace amounts of mycobacterial DNA. PCR amplifications and DNA sequencing results showed that the $M$. ulcerans IS2404 was detected in WGA-amplified DNA from a crayfish that was collected in summer (but not in the crayfish collected in autumn or in any other sample), presenting so far the only piece of evidence that links a contaminated aquatic environment to BU occurrence in Japan.

Intriguingly, $M$. ulcerans IS2404 was detected in a crayfish but not in the water samples collected from the same channel at the same time [47], indicating that despite the presence of the pathogen in the contaminated aquatic environment, its potential presence in the water was nevertheless below the detection sensitivity of PCR amplification. In an aquatic community, due to biotic interactions between various habitants such as filter feeders, herbivorous, scavengers, and predators, $M$. ulcerans may be widespread across the whole community. In agreement with this point of view, in endemic areas $M$. ulcerans DNA has been detected in a wide variety of environment samples, such as aquatic insects, biofilms, soil, mosquitoes, crustaceans, detritus, fish, frogs, snails, worms, and various small mammals [49]. The transmission of pathogens within an aquatic environment, depending on biodiversity and the number of habitants, can drive the environmental load of the pathogens to be enriched or diluted in certain hosts, carriers, or biofilms. Additionally, certain keystone organisms could play an overwhelming role in the transmission and overall prevalence of $M$. ulcerans in a specific environment [50]. The crayfish (Procambarus clarkii) is known to have a wide food range, including plants, small 
fish, shrimp, plankton, benthos, and algae, and especially prefers mud and deceased aquatic organisms. Such an omnivorous eating habit may explain an enrichment of M. ulcerans in crayfish.

\subsection{Seasonal Variation of BU in Japan}

In addition to establishing the presence of $M$. ulcerans subsp. shinshuense in an aquatic environment in Japan for the first time, the findings of the field work were in line with a noticeable feature of seasonal variation of BU occurrence in Japan. Eighty percent of BU cases in Japan were diagnosed in autumn and winter (from September to February), but only a few in summer (July and August) $[6,8]$. Under the assumption that the diagnosis is preceded for several months by $M$. ulcerans infection due to a long incubation period [9] and due to the delay in seeking medical care, it was speculated that the exposure to the pathogen occurred during summer. Such dynamics in M. ulcerans infection are putatively driven by a complex interplay of human activities and the prevalence of the pathogen in the environment. Japan has four distinct seasons with a rainy season throughout early summer (typically from early June to mid-July), and there are naturally more chances for bare skin to be exposed to an aquatic environment during the hot and humid summer time, especially in the water-rich countryside.

On the other hand, there was no direct information regarding the prevalence of M. ulcerans in the environment throughout the year in Japan before the field work. The results that $M$. ulcerans subsp. shinshuense was detected only in July, but not in any of the samples collected from the same water channel in October, hints at potential seasonally changing prevalence of environmental M. ulcerans subsp. shinshuense with a high peak in summer, which may have contributed to the observed seasonal variation of BU incidence in Japan. However, the scale of this fieldwork alone was far too small to make a solid prediction. Seasonal dynamics in environmental $M$. ulcerans prevalence can be driven by an extremely complex interplay between multiple biotic and abiotic factors, such as topographic factors (distribution and latitude of land cover of watersheds), climatic factors (temperature, precipitation), physical-chemical conditions ( $\mathrm{pH}$, dissolved oxygen, salinity) in stagnant and slow flowing ecosystems, and networks of aquatic taxa, as suggested by large-scale field studies conducted in BU endemic countries [51, 52]. In this respect, the seasonal dynamics of the prevalence of M. ulcerans in Japan (especially in a high latitude area as the site in the field work) may differ substantially from that reported in tropical/subtropical areas.

\subsection{PCR Detection of $M$. ulcerans subsp. shinshuense DNA in the Environment}

Field studies to detect environmental M. ulcerans subsp. shinshuense in Japan are still in their infancy compared to studies in BU endemic countries. Presently, the detection of $M$. ulcerans in the environment is mostly based on demonstrating by 
PCR the presence of IS2404, an insertion sequence with over 200 copies in the whole genome of M. ulcerans [10]. IS2404-PCR is highly specific and sensitive for testing clinical specimens from human BU lesions; however, its application in the detection of environmental M. ulcerans is less straightforward, and the IS2404PCR-based results should be interpreted with caution. IS2404-PCR detects the presence of IS2404 regardless of the pathogenicity of the detected mycobacteria, which alone has little power in predicting endemicity of BU. M. ulcerans DNA was detected by IS2404-PCR extensively across southern Louisiana, U.S., leading to the conclusion that the distribution of environmental M. ulcerans is not restricted to areas where BU is endemic [53].

In a large-scale field study conducted in Benin, IS2404-positive samples were detected with similar prevalence in environmental samples in both non-endemic (9/10) and endemic (12/12) villages [49]. However, when IS2404-positive samples were further analyzed for a second PCR target, the enoyl reductase (ER) that is required for the synthesis of mycolactone, only 2/10 non-endemic villages had ER-positive samples, whereas 9/12 endemic villages had ER-positive samples. Thus, the PCR for IS2404 alone falsely predicted 9/10 non-endemic villages to be endemic, and the additional use of the PCR for ER accurately predicted 8/10 nonendemic villages [49]. In a field study, the use of a single PCR target for detection of environmental $M$. ulcerans may easily signal a false alarm to public health authorities. Secondly, the results of conventional IS2404 PCR analyses are only qualitative and do not reveal the abundance of the pathogen. They are thus inadequate to describe potential seasonal dynamics in the overall environmental load of M. ulcerans. Lastly, analysis of many IS2404-positive samples in Ghana have revealed the presence of mycolactone-producing mycobacterial species other than the ones commonly associated with BU disease in humans [54]. Mycobacterial strains closely related to the human pathogenic M. ulcerans, including ecovars originally designated $M$. liflandii, M. pseudoshottsii, and mycolactoneproducing $M$. marinum, have been found to harbor IS2404 [55, 56].

Thus, the analyses of M. ulcerans that rely solely upon the conventional PCR assay for IS2404 are flawed. TaqMan Multiplex real-time PCR assays that target two insertion sequences of $M$. ulcerans (IS2404, IS2606) and a multicopy sequence encoding the ketoreductase B domain $[55,57]$, in which samples are considered positive only if the detected cycle threshold values are strictly lower than a default value based on external standard curves with serial dilutions of Mycobacterial DNA, can quantify the copy number of the targets to allow the differentiation of classical M. ulcerans from other IS2404-harboring strains in the environment [55, 57].

Moreover, a novel category of variable tandem repeats (VNTRs) called mycobacterial interspersed repetitive units (MIRUs) have been identified in the genomes of $M$. ulcerans and M. marinum. Analysis of strain-specific polymorphisms of MIRU loci has been used to tentatively genotype $M$. ulcerans, $M$. marinum, and an M. marinum-like organism that is considered a possible missing link between $M$. ulcerans and $M$. marinum $[56,58]$. Ideas inspired by many studies conducted in the BU endemic countries, such as the use of multiple targets in quantitative PCR assays and MIRU-VNTR typing, will better equip us to evaluate the environmental 
distribution of M. ulcerans in future field studies in Japan. Distribution maps of Asian strains will play a key role in understanding the transmission mode of $M$. ulcerans and support control of BU.

\section{$4 \quad$ BU in Asia and Future Perspectives}

\subsection{BU in China}

In addition to BU cases reported from Japan, M. ulcerans subsp. shinshuense has also been identified as the causative agent of BU in a patient from China [59]. In 1997, a 40-year-old women, who had grown up in China and had been living in Europe, traveled to Shandong Province, China in the summer (July and August). According to the patient's narrative, she received many mosquito bites after walking barelegged in the field. Three months later, the patient noticed a swelling at the front of her left lower leg that afterwards developed a slightly depressed center with no other systemic symptoms. Four months after that, a surgeon excised her lesion. Reportedly, there was no noticeable bleeding during the procedure, and the excised tissue was pale and firm. The lesion did not heal after excision but further developed ulceration. After the patient went back to Europe, a doctor specializing in infectious diseases examined the lesion and suggested that it might be skin tuberculosis. Consequently, a smear of the ulcer base was made by the patient's general practitioner, and Ziehl-Neelsen staining revealed acid-fast bacteria scattered and in clumps. The patient was therefore referred to the Department of Dermatology, Section of Tropical Dermatology of the Academic Medical Center, Amsterdam, where diagnostic procedures showed BU-characteristic clinical, histopathological, and molecular findings [59].

At first examination, it was a single painless lesion $(3 \times 3.5 \mathrm{~cm}$ in diameter $)$ on the lower leg with necrotic ulcer bed and undermined border. Histopathological examination of the biopsy specimens showed thickening and broadening of the epidermis, extensive eosinophilic necrosis in the deeper dermis and the subcutaneous tissue without granulomatous inflammatory reactions. Cultivation tests using a biopsy sample yielded pale-cream to yellow colonies after 40-day incubation on Coletsos medium only at $30^{\circ} \mathrm{C}$. PCR analyses using DNA extracts both from ulcer biopsies and culture isolates confirmed the presence of the M. ulcerans-specific IS2404 sequence. Using classical identification schemes the isolate was identified as M. ulcerans subsp. shinshuense. Treatment began with RFP + CAM and was later switched to ciprofloxacin $(\mathrm{CPFX})+$ rifabutin $(\mathrm{RFB})$ based on the results from drug susceptibility test of the original culture isolate. The patient was cured with an 8-months antibiotic treatment without reported recurrence [59].

The putative infection site of Shandong Province, China is above latitude $30^{\circ} \mathrm{N}$. BU occurrence at such a high latitude is rare, and had only been reported in Japan, suggesting that $M$. ulcerans subsp. shinshuense is more adapted to environments at higher latitudes than the classical $M$. ulcerans that is observed to be confined to tropical/subtropical regions and temperate climatic areas. Moreover, the infection by M. ulcerans subsp. shinshuense in the patient from China was highly 
suspected to have occurred during her travel in summer, 3-4 months prior to symptom onset, which is in line with the proposed seasonal variation of BU occurrence in Japan. Thus, in contrast to the majority of BU cases that are caused by classical M. ulcerans, BU infections by M. ulcerans subsp. shinshuense have so far been only infrequently found in Japan and China, as apparent from the clinical reports $[6,8]$.

As stated before, it is possible that extensive reductive genomic changes have enabled the classical lineage to habituate in a more confined environment, resulting in clonal populations within Africa and Australia with genomic rearrangements rendering them more pathogenic and strengthening their ability to infect mammals [15]. In contrast, the ancestral lineage, closer to the $M$. marinum progenitor, may have remained as a largely environmental mycobacterium that only occasionally affects humans. From this point of view, it is reasonable to predict that large-scale field studies in Japan or China may reveal an unexpectedly extensive presence of $M$. ulcerans subsp. shinshuense in the environment that does not necessarily lead to an outbreak of BU.

\subsection{Toward Detection of BU in Other Asian Countries}

It appears that the stably increased number of reported BU cases from Japan is due to increased awareness of the disease among health workers rather than to an actual rise in the number of infections. On the other hand, the extremely low disease reporting rate in China and the absence of BU reporting from the neighboring Asian countries may be indicative of hidden cases. In non-endemic and less resourced Asian countries, awareness and knowledge of BU disease among health workers and the community are lacking, so there is the possibility of misdiagnosis. Moreover, insufficient health infrastructure and geographical challenges can also contribute to the underreporting of cases. Despite little evidence supporting a predictable future outbreak of BU in Asia, the importance of an early diagnosis cannot be overemphasized because of the potential life-long disfigurement and disabilities this disease can cause if left undiagnosed and untreated. Residents of Asian countries should be aware that $\mathrm{BU}$ is a mycobacterial infection that is not limited to tropical or subtropical regions.

Education of health workers, in particular dermatologists, in Asian countries is essential for early diagnosis of BU. Also, it is important to provide public health education to familiarize people, especially those living near rural aquatic environments, with BU symptoms. Since international travel has become more popular, the so-called imported tropical diseases may be seen more frequently than before. In addition to early diagnosis, the establishment of a standard treatment guideline optimized for BU infections in Asian countries is also needed to ensure efficacy without unnecessary surgery and overtreatment. Furthermore, a comprehensive comparison of the proteomes of $M$. ulcerans subsp. shinshuense with classical M. ulcerans may shed new light on the differences in their adaptive biology and response to antibiotic treatment.

For investigating the phylogenetics of $M$. ulcerans subsp. shinshuense, various approaches including genomic and biochemical analyses have been carried out $[2,4,7,12,13,15]$. Only one strain of M. ulcerans subsp. shinshuense has 
undergone whole genome sequencing so far [13]. Genomic differences among the sequenced strain of $M$. ulcerans subsp. shinshuense and other ecovars of M. ulcerans have been found. Further comparative genomic analyses with multiple $M$. ulcerans subsp. shinshuense strains, including the Chinese strain, would enable a more precise phylogenetic characterization of M. ulcerans subsp. shinshuense, which could give information on the spread of these bacteria in Japan as well as in China. However, at the moment, only one Chinese strain has been recognized, so discovery of multiple Chinese strains might further contribute to the above-mentioned phylogenetic resolution. Additionally, it is still unclear whether the clinical features caused by $M$. ulcerans subsp. shinshuense differ from those of other M. ulcerans strains. Future attempts involving generation of recombinant and mutant strains of M. ulcerans subsp. shinshuense might clarify the unique characteristics of this $M$. ulcerans subspecies and could provide important clues into the pathogenic mechanisms of BU.

Acknowledgements The authors would like to thank Ms. Kayo Shinozaki for her assistance to prepare the manuscript.

\section{References}

1. Mikoshiba H, Shindo Y, Matsumoto H et al (1982) A case of typical mycobacteriosis due to Mycobacterium ulcerans-like organism (in Japanese). Jpn J Dermatol 92(5):557-565

2. Tsukamura M, Kaneda K, Imaeda $T$ et al (1989) A taxonomic study on a mycobacterium which caused a skin ulcer in a Japanese girl and resembled Mycobacterium ulcerans. Kekkaku 64(11):691-697

3. Kazumi Y, Ohtomo K, Takahashi M et al (2004) Mycobacterium shinshuense isolated from cutaneous ulcer lesion of right lower extremity in a 37-year-old woman (in Japanese). Kekkaku 79(7):437-441

4. Nakanaga K, Ishii N, Suzuki K et al (2007) "Mycobacterium ulcerans subsp. shinshuense" isolated from a skin ulcer lesion: identification based on 16S rRNA gene sequencing. J Clin Microbiol 45(11):3840-3843. https://doi.org/10.1128/JCM.01041-07

5. Yotsu RR, Nakanaga K, Hoshino Y et al (2012) Buruli ulcer and current situation in Japan: a new emerging cutaneous Mycobacterium infection. J Dermatol 39(7):587-593. https://doi. org/10.1111/j.1346-8138.2012.01543.x

6. Yotsu RR, Murase C, Sugawara M et al (2015) Revisiting Buruli ulcer. J Dermatol 42(11):10331041. https://doi.org/10.1111/1346-8138.13049

7. Nakanaga K, Hoshino Y, Yotsu RR et al (2011) Nineteen cases of Buruli ulcer diagnosed in Japan from 1980 to 2010. J Clin Microbiol 49(11):3829-3836. https://doi.org/10.1128/ JCM.00783-11

8. Sugawara M, Ishii N, Nakanaga K et al (2015) Exploration of a standard treatment for Buruli ulcer through a comprehensive analysis of all cases diagnosed in Japan. J Dermatol 42(6):588595. https://doi.org/10.1111/1346-8138.12851

9. Trubiano JA, Lavender CJ, Fyfe JA et al (2013) The incubation period of Buruli ulcer (Mycobacterium ulcerans infection). PLoS Negl Trop Dis 7(10):e2463. https://doi.org/10.1371/ journal.pntd.0002463

10. Stinear TP, Seemann T, Pidot S et al (2007) Reductive evolution and niche adaptation inferred from the genome of Mycobacterium ulcerans, the causative agent of Buruli ulcer. Genome Res 17(2):192-200. https://doi.org/10.1101/gr.5942807 
11. Tobias NJ, Doig KD, Medema MH et al (2013) Complete genome sequence of the frog pathogen Mycobacterium ulcerans ecovar Liflandii. J Bacteriol 195(3):556-564. https://doi. org/10.1128/JB.02132-12

12. Pidot SJ, Hong H, Seemann T et al (2008) Deciphering the genetic basis for polyketide variation among mycobacteria producing mycolactones. BMC Genomics 9:462. https://doi. org/10.1186/1471-2164-9-462

13. Yoshida M, Nakanaga K, Ogura Y et al (2016) Complete genome sequence of Mycobacterium ulcerans subsp. shinshuense. Genome Announc 4(5):e01050-16. https://doi.org/10.1128/ genomeA.01050-16

14. Arora G, Sajid A, Singhal A et al (2014) Identification of Ser/Thr kinase and forkhead associated domains in Mycobacterium ulcerans: characterization of novel association between protein kinase Q and MupFHA. PLoS Negl Trop Dis 8(11):e3315. https://doi.org/10.1371/ journal.pntd.0003315

15. Kaser M, Rondini S, Naegeli M et al (2007) Evolution of two distinct phylogenetic lineages of the emerging human pathogen Mycobacterium ulcerans. BMC Evol Biol 7:177. https://doi. org/10.1186/1471-2148-7-177

16. Qi W, Kaser M, Roltgen K et al (2009) Genomic diversity and evolution of Mycobacterium ulcerans revealed by next-generation sequencing. PLoS Pathog 5(9):e1000580. https://doi. org/10.1371/journal.ppat.1000580

17. George KM, Chatterjee D, Gunawardana G et al (1999) Mycolactone: a polyketide toxin from Mycobacterium ulcerans required for virulence. Science 283(5403):854-857

18. Fidanze S, Song F, Szlosek-Pinaud M et al (2001) Complete structure of the mycolactones. J Am Chem Soc 123(41):10117-10118

19. Ranger BS, Mahrous EA, Mosi L et al (2006) Globally distributed mycobacterial fish pathogens produce a novel plasmid-encoded toxic macrolide, mycolactone F. Infect Immun 74(11):6037-6045. https://doi.org/10.1128/IAI.00970-06

20. Mve-Obiang A, Lee RE, Umstot ES et al (2005) A newly discovered mycobacterial pathogen isolated from laboratory colonies of Xenopus species with lethal infections produces a novel form of mycolactone, the Mycobacterium ulcerans macrolide toxin. Infect Immun 73(6):3307-3312. https://doi.org/10.1128/IAI.73.6.3307-3312.2005

21. Hong H, Spencer JB, Porter JL et al (2005) A novel mycolactone from a clinical isolate of Mycobacterium ulcerans provides evidence for additional toxin heterogeneity as a result of specific changes in the modular polyketide synthase. Chembiochem 6(4):643-648. https://doi. org/10.1002/cbic.200400339

22. Brennan PJ, Nikaido H (1995) The envelope of mycobacteria. Annu Rev Biochem 64:29-63. https://doi.org/10.1146/annurev.bi.64.070195.000333

23. Daffe M, Draper P (1998) The envelope layers of mycobacteria with reference to their pathogenicity. Adv Microb Physiol 39:131-203

24. Hunter SW, Brennan PJ (1981) A novel phenolic glycolipid from Mycobacterium leprae possibly involved in immunogenicity and pathogenicity. J Bacteriol 147(3):728-735

25. Daffe M, Lacave C, Laneelle MA et al (1987) Structure of the major triglycosyl phenol-phthiocerol of Mycobacterium tuberculosis (strain Canetti). Eur J Biochem 167(1): $155-160$

26. Dobson G, Minnikin DE, Besra GS et al (1990) Characterisation of phenolic glycolipids from Mycobacterium marinum. Biochim Biophys Acta 1042(2):176-181

27. Daffe M, Varnerot A, Levy-Frebault VV (1992) The phenolic mycoside of Mycobacterium ulcerans: structure and taxonomic implications. J Gen Microbiol 138(1):131-137. https://doi. org/10.1099/00221287-138-1-131

28. Cho SN, Yanagihara DL, Hunter SW et al (1983) Serological specificity of phenolic glycolipid I from Mycobacterium leprae and use in serodiagnosis of leprosy. Infect Immun 41(3):1077-1083

29. Ng V, Zanazzi G, Timpl R et al (2000) Role of the cell wall phenolic glycolipid-1 in the peripheral nerve predilection of Mycobacterium leprae. Cell 103(3):511-524 
30. Reed MB, Domenech P, Manca C et al (2004) A glycolipid of hypervirulent tuberculosis strains that inhibits the innate immune response. Nature 431(7004):84-87. https://doi.org/10.1038/ nature 02837

31. Grode L, Seiler P, Baumann S et al (2005) Increased vaccine efficacy against tuberculosis of recombinant Mycobacterium bovis bacille Calmette-Guerin mutants that secrete listeriolysin. J Clin Invest 115(9):2472-2479. https://doi.org/10.1172/JCI24617

32. Lin W, Mathys V, Ang EL et al (2012) Urease activity represents an alternative pathway for Mycobacterium tuberculosis nitrogen metabolism. Infect Immun 80(8):2771-2779. https:// doi.org/10.1128/IAI.06195-11

33. Benedetti MS (1995) Inducing properties of rifabutin, and effects on the pharmacokinetics and metabolism of concomitant drugs. Pharmacol Res 32(4):177-187

34. Chen J, Raymond K (2006) Roles of rifampicin in drug-drug interactions: underlying molecular mechanisms involving the nuclear pregnane X receptor. Ann Clin Microbiol Antimicrob 5:3. https://doi.org/10.1186/1476-0711-5-3

35. Alffenaar JW, Nienhuis WA, de Velde F et al (2010) Pharmacokinetics of rifampin and clarithromycin in patients treated for Mycobacterium ulcerans infection. Antimicrob Agents Chemother 54(9):3878-3883. https://doi.org/10.1128/AAC.00099-10

36. Wallace RJ Jr, Brown BA, Griffith DE et al (1995) Reduced serum levels of clarithromycin in patients treated with multidrug regimens including rifampin or rifabutin for Mycobacterium avium-M. intracellulare infection. J Infect Dis 171(3):747-750

37. World Health Organization (2015) WHO meeting on Buruli ulcer 2015 abstract. WHO, Geneva

38. Converse PJ, Nuermberger EL, Almeida DV et al (2011) Treating Mycobacterium ulcerans disease (Buruli ulcer): from surgery to antibiotics, is the pill mightier than the knife? Future Microbiol 6(10):1185-1198. https://doi.org/10.2217/fmb.11.101

39. Murase C, Kono M, Nakanaga K et al (2015) Buruli ulcer successfully treated with negativepressure wound therapy. JAMA Dermatol 151(10):1137-1139. https://doi.org/10.1001/ jamadermatol.2015.1567

40. Rosul MV, Patskan BM (2016) Ozone therapy effectiveness in patients with ulcerous lesions due to diabetes mellitus. Wiad Lek 69(1):7-9

41. Bertolottim A, Izzo A, Grigolato P et al (2013) The use of ozone therapy in Buruli ulcer had an excellent outcome. BMJ Case Rep 2013:bcr2012008249. https://doi.org/10.1136/ bcr-2012-008249

42. Perdrizet GA (2014) Principles and practice of hyperbaric medicine: a medical practitioner's primer, part II. Conn Med 78(7):389-402

43. Dauwe P, Pulikkottil B, Lavery L et al (2014) Does hyperbaric oxygen therapy work in facilitating acute wound healing: a systematic review. Plast Reconstr Surg 133(2):208e-215e. https://doi.org/10.1097/01.prs.0000436849.79161.a4

44. Krieg RE, Wolcott JH, Confer A (1975) Treatment of Mycobacterium ulcerans infection by hyperbaric oxygenation. Aviat Space Environ Med 46(10):1241-1245

45. Pszolla N, Sarkar MR, Strecker W et al (2003) Buruli ulcer: a systemic disease. Clin Infect Dis 37(6):e78-e82. https://doi.org/10.1086/377170

46. Krieg RE, Wolcott JH, Meyers WM (1979) Mycobacterium ulcerans infection: treatment with rifampin, hyperbaric oxygenation, and heat. Aviat Space Environ Med 50(9):888-892

47. Luo Y, Degang Y, Ohtsuka M et al (2015) Detection of Mycobacterium ulcerans subsp. shinshuense DNA from a water channel in familial Buruli ulcer cases in Japan. Future Microbiol 10(4):461-469. https://doi.org/10.2217/fmb.14.152

48. Ohtsuka M, Kikuchi N, Yamamoto T et al (2014) Buruli ulcer caused by Mycobacterium ulcerans subsp. shinshuense: a rare case of familial concurrent occurrence and detection of insertion sequence 2404 in Japan. JAMA Dermatol 150(1):64-67. https://doi.org/10.1001/ jamadermatol.2013.6816

49. Williamson HR, Benbow ME, Campbell LP et al (2012) Detection of Mycobacterium ulcerans in the environment predicts prevalence of Buruli ulcer in Benin. PLoS Negl Trop Dis 6(1):e1506. https://doi.org/10.1371/journal.pntd.0001506 
50. Roche B, Benbow ME, Merritt R et al (2013) Identifying the Achilles' heel of multi-host pathogens: the concept of keystone "host" species illustrated by Mycobacterium ulcerans transmission. Environ Res Lett 8(4):045009. https://doi.org/10.1088/1748-9326/8/4/045009

51. Garchitorena A, Guegan JF, Leger L et al (2015) Mycobacterium ulcerans dynamics in aquatic ecosystems are driven by a complex interplay of abiotic and biotic factors. Elife 4:e07616. https://doi.org/10.7554/eLife.07616

52. Garchitorena A, Ngonghala CN, Texier G et al (2015) Environmental transmission of Mycobacterium ulcerans drives dynamics of Buruli ulcer in endemic regions of Cameroon. Sci Rep 5:18055. https://doi.org/10.1038/srep18055

53. Hennigan CE, Myers L, Ferris MJ (2013) Environmental distribution and seasonal prevalence of Mycobacterium ulcerans in Southern Louisiana. Appl Environ Microbiol 79(8):2648-2656. https://doi.org/10.1128/AEM.03543-12

54. Williamson HR, Benbow ME, Nguyen KD et al (2008) Distribution of Mycobacterium ulcerans in Buruli ulcer endemic and non-endemic aquatic sites in Ghana. PLoS Negl Trop Dis 2(3):e205. https://doi.org/10.1371/journal.pntd.0000205

55. Fyfe JA, Lavender CJ, Johnson PD et al (2007) Development and application of two multiplex real-time PCR assays for the detection of Mycobacterium ulcerans in clinical and environmental samples. Appl Environ Microbiol 73(15):4733-4740. https://doi.org/10.1128/ AEM.02971-06

56. Stragier P, Ablordey A, Durnez L et al (2007) VNTR analysis differentiates Mycobacterium ulcerans and IS2404 positive mycobacteria. Syst Appl Microbiol 30(7):525-530. https://doi. org/10.1016/j.syapm.2007.06.001

57. Garchitorena A, Roche B, Kamgang R et al (2014) Mycobacterium ulcerans ecological dynamics and its association with freshwater ecosystems and aquatic communities: results from a 12-month environmental survey in Cameroon. PLoS Negl Trop Dis 8(5):e2879. https:// doi.org/10.1371/journal.pntd.0002879

58. Stragier P, Ablordey A, Meyers WM et al (2005) Genotyping Mycobacterium ulcerans and Mycobacterium marinum by using mycobacterial interspersed repetitive units. J Bacteriol 187(5):1639-1647. https://doi.org/10.1128/JB.187.5.1639-1647.2005

59. Faber WR, Arias-Bouda LM, Zeegelaar JE et al (2000) First reported case of Mycobacterium ulcerans infection in a patient from China. Trans R Soc Trop Med Hyg 94(3):277-279

Open Access This chapter is licensed under the terms of the Creative Commons Attribution 4.0 International License (http://creativecommons.org/licenses/by/4.0/), which permits use, sharing, adaptation, distribution and reproduction in any medium or format, as long as you give appropriate credit to the original author(s) and the source, provide a link to the Creative Commons license and indicate if changes were made.

The images or other third party material in this chapter are included in the chapter's Creative Commons license, unless indicated otherwise in a credit line to the material. If material is not included in the chapter's Creative Commons license and your intended use is not permitted by statutory regulation or exceeds the permitted use, you will need to obtain permission directly from the copyright holder.

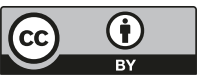

